\title{
Compressed RSS Measurement for Communication and Sensing in the Internet of Things
}

\author{
Yanchao Zhao, ${ }^{1,2,3}$ Wenzhong Li, ${ }^{2}$ Jie $\mathrm{Wu}^{4}{ }^{4}$ Sanglu $\mathrm{Lu},{ }^{2,3}$ and Bing Chen ${ }^{1,3}$ \\ ${ }^{1}$ College of Computer Science and Technology, Nanjing University of Aeronautics and Astronautics, Nanjing, China \\ ${ }^{2}$ State Key Laboratory for Novel Software Technology, Nanjing University, Nanjing, China \\ ${ }^{3}$ Collaborative Innovation Center of Novel Software Technology and Industrialization, Nanjing, China \\ ${ }^{4}$ Department of Computer and Information Sciences, Temple University, Philadelphia, PA, USA
}

Correspondence should be addressed to Yanchao Zhao; yczhao@nuaa.edu.cn

Received 29 April 2017; Accepted 6 July 2017; Published 7 August 2017

Academic Editor: Feng Wang

Copyright (c) 2017 Yanchao Zhao et al. This is an open access article distributed under the Creative Commons Attribution License, which permits unrestricted use, distribution, and reproduction in any medium, provided the original work is properly cited.

\begin{abstract}
The receiving signal strength (RSS) is crucial for the Internet of Things (IoT), as it is the key foundation for communication resource allocation, localization, interference management, sensing, and so on. Aside from its significance, the measurement process could be tedious, time consuming, inaccurate, and involving human operations. The state-of-the-art works usually applied the fashion of "measure a few, predict many," which use measurement calibrated models to generate the RSS for the whole networks. However, this kind of methods still cannot provide accurate results in a short duration with low measurement cost. In addition, they also require careful scheduling of the measurement which is vulnerable to measurement conflict. In this paper, we propose a compressive sensing- (CS-) based RSS measurement solution, which is conflict-tolerant, time-efficient, and accuracyguaranteed without any model-calibrate operation. The CS-based solution takes advantage of compressive sensing theory to enable simultaneous measurement in the same channel, which reduces the time cost to the level of $\mathcal{O}(\log N)$ (where $N$ is the network size) and works well for sparse networks. Extensive experiments based on real data trace are conducted to show the efficiency of the proposed solutions.
\end{abstract}

\section{Introduction}

With the ubiquitous wireless networking devices, we envision realizing the Internet of Things, which requires the wireless networks to develop with higher spectrum utilization, less transmission delay, and lower energy consumption. This trend gives birth to many emerging technologies (e.g., OFDMA, network coding, and cognitive radio [1-3]). To realize the IoT, a key job is how to optimize the allocation of existing wireless communication resources (e.g., link scheduling, channel allocation, and power allocation $[4,5])$, improve the communication bandwidth, and reduce the communication power consumption. Aside from the traditional usage of data transmission, recent research efforts even extend the usage of the wireless signal in IoT to perform localization [6] and activity recognition [7]. All of them, although used differently, rely on the receiving signal strength (RSS) and its variant. Specifically, for communication resource allocations, it requires the RSS to build the interference models. Nevertheless, for localization in wireless networks, it requires the RSS to compute the distance or to generate the fingerprint of certain location. Thus, the accuracy of the measured RSS will finally affect the optimization results as well as the localization accuracy. The efficiency of the measurement process will also affect the applicability and efficiency of these wireless applications.

Towards the efficient and accurate RSS measurement and its application in resource allocation and localization, most existing works [8-10] mainly focus on how to derive the metric (e.g., SINR and RSS fingerprint) by some signal propagation models (e.g., the path loss model [10]) with fixed empirical parameters. However, such propagation models and the corresponding empirical parameters cannot characterize the complex, time-varying channel conditions 


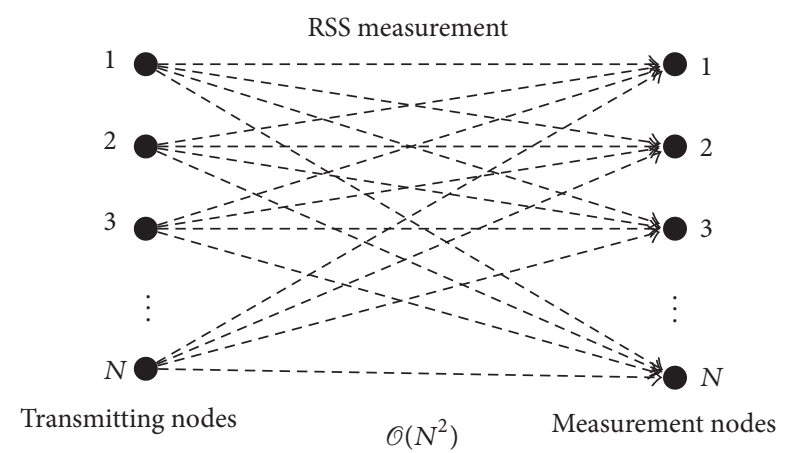

FIGURE 1: Traditional exhaustive RSS measurement.

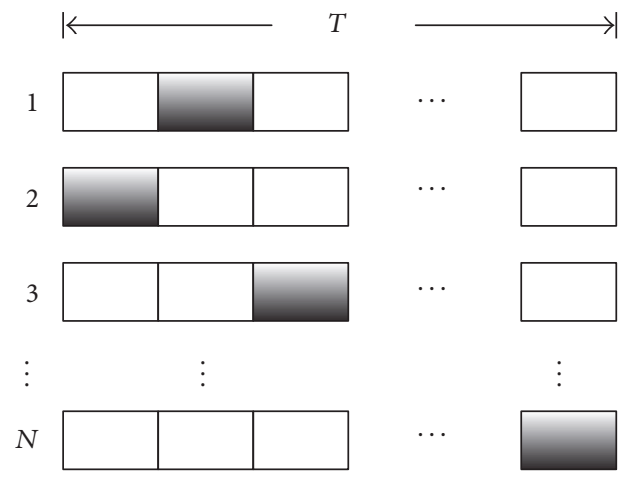

FIGURE 2: Nonconflict RSS measurement.

accurately, which in turn compromise the optimization and localization results.

To obtain accurate RSS values, exhausting measurement on all wireless links will incur unacceptable time cost. First, the RSS collection process, for example, the SINRoptimization process, requires the RSS values between every pair of nodes in the network, so the number of RSS values to be measured grows quadratically with the network size. As is illustrated in Figure 1, the left row and right row of the nodes are the same set of nodes in the network, while the links between the nodes represent the measurement conducted between different nodes. The RSS measurements should be conducted in every pair of nodes, thus leading to a measurement cost in level of $\mathcal{O}\left(N^{2}\right)$. Meanwhile, to measure the RSS accurately, it usually requires that the receiving signal is decodable, such that the transmitting node could be identified with the source information recoded in the packet. As is illustrated in Figure 2, no measurement should be performed simultaneously in the process of RSS measurement, because the conflict will lead to two major drawbacks. First, the conflict of two signals could cause the failure of packet decoding, so that the sources of the signals could not be identified. Secondly, even the packet could be decoded when the capture effect exists; the measured RSS will be the overlapping one of both signals; thus, the measured RSS will be far away from the intended one. Due to the above reasons, traditional RSS measurements, as illustrated in Figure 2, where the grey grid stands for the slot used for measurements, were performed in a nonconflicting way. This

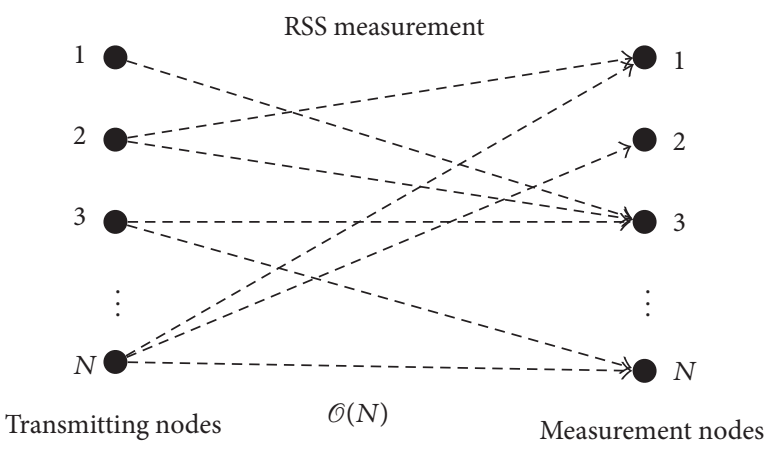

FIGURE 3: Compressive sensing-based RSS measurement.

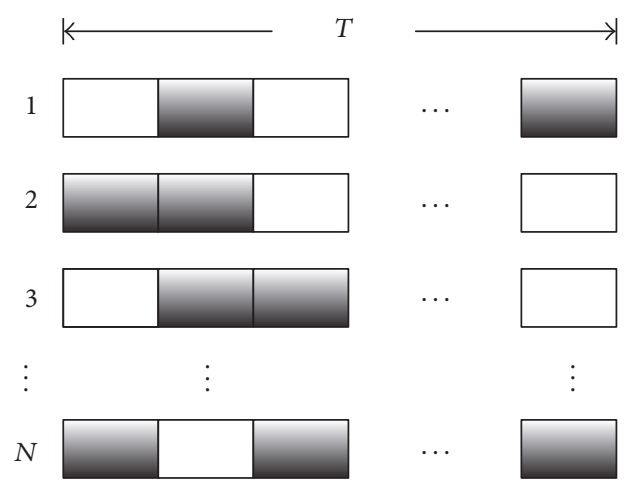

FIGURE 4: Simultaneous RSS measurement.

kind of measurement method makes the time consumption of whole measurement process up to the level of $\mathcal{O}\left(N^{2}\right)$.

Both the measurement cost and the time consumption are unacceptable for a network with large size of nodes. To tackle this, we propose a compressive sensing-based solution, whose basic idea could be summarized as two aspects: partial measurement and simultaneous measurement.

The idea of partial measurement is illustrated in Figure 3. As in the figure, only a subset of node pairs is selected to measure the RSS. The number of measured links could be as few as $\mathcal{O}(N)$ or even decreased to the level of $\mathcal{O}(\log N)$, which is achieved by the method proposed in this paper. Based on the measured RSS, the other unknown RSS values could be estimated with certain accuracy. In summary, the partial measurement method is based on the concept of "measure a few, predict many."

The idea of simultaneous measurement is illustrated in Figure 4, where $T$ is the total time used, while the grids colored with grey imply that slots are used for measurement. It clearly presents the advantage that the total measurement time could be deducted. However, as aforementioned, the simultaneous measurement is forbidden, as we cannot tell two overlapped signals apart. In turn, we also cannot get the values of RSS and do not know where is the signal coming from. However, by utilizing the nature of linear additivity of RSS, we show how to distinguish the source of the RSS without assuming that the signal could be decoded and tell the values of the RSS. 
The problems associated with the partial measurement and simultaneous measurement could be jointly solved in our proposed compressive sensing-based solution, which mainly introduce the idea of compressive sensing and lead to the measurement cost and time cost to the level of $\mathcal{O}(\log N)$. The CS-based solution allows simultaneous measurements on a single channel. This is mainly empowered by the development of compressive sensing theory [11-13]. The efficiency is achieved by the result of the CS theory that not enough overlapping measurements ( $N$ dimensions require $N$ sets of measurements) can recover the original matrix (in our case it refers to the RSS matrix). It further reduces the time cost to the level of $\mathcal{O}(\log N)$, which highly outperforms the traditional solutions. However, it only achieves acceptable accuracy in sparse networks. The accuracy is guaranteed by controlling the number of measurements and the sparsity of the RSS matrix. A number of theories are established to study the relationship between the accuracy and the number of measurements [13]. A measurement matrix generation method and a sparsity control method are proposed to address the specific issues of CS-based measurement problem. lows:

The contributions of this paper are summarized as fol-

(i) We reveal the important problem of accurate and efficient RSS measurement for communication and sensing in IoT.

(ii) We modeled the RSS measurement process as a linear system and propose a basic framework to perform simultaneous measurement of RSS instead of the traditional nonconflict measurement.

(iii) We further present a compressive sensing- (CS-) based solution to achieve partial measurement. It can achieve the time efficiency of $\mathcal{O}(\log N)$ with accuracy control. Both centralized and distributed solutions are proposed.

(iv) We introduce a LDPC-based measurement matrix, which only generates a small number of measurements. It greatly reduces the energy consumption for IoT.

(v) We conduct extensive experiments using real communication traces collected from a wireless mesh network testbed, which show the efficiency of the proposed solutions.

The rest of this paper is organized as follows. Section 5 introduces related work. The system model and problem definition are presented in Section 2.1. The CS-based solution is presented in Section 3. The numerical results are illustrated in Section 4. Finally, the paper is concluded in Section 6.

\section{Preliminaries}

In this section, we explore the first step to achieve partial measurement and simultaneous measurement, which is to model the efficient RSS measurement problem as a linear system. Before proposing the modeling, we first propose the network model and some important metrics to evaluate our solution.

2.1. Network Model. We consider a synchronized, timeslotted wireless network consisting of $N$ nodes denoted by $\mathcal{N}$. A set of channels, denoted by $\mathscr{M}$, is available to each node in $\mathcal{N}$. We denote $P$ as the sending power of each node operating over any channel and by $p_{i j}^{m}$ as the RSS of a signal from node $i \in \mathcal{N}$ over channel $m \in \mathscr{M}$ received at node $j \in \mathscr{N}$.

Our main task is to obtain all the RSS values over each pair of nodes and each channel: that is,

$$
\left\{p_{i j}^{m} \mid i, j \in \mathcal{N}, i \neq j, m \in \mathscr{M}\right\} .
$$

A measurement scheme could be evaluated via the following metrics:

(i) Time cost: it is the total time slots to accomplish the measurement process.

(ii) Overhead: it is the total number of measurements in all nodes and channels.

(iii) Accuracy: we defined two levels of accuracy, which are link-wise accuracy and network-wide accuracy, receptively. Regarding link-wise accuracy, the result of measurement should be within a certain level of confidence $1-\alpha / 2$. Regarding the network-wide accuracy, it implies that $\beta$ portion of $\left\{p_{i j}^{m}\right\}$ is accurate. The CS-based solution only achieves network-wide accuracy.

It is worth mentioning that the relation between link-wise accuracy and network-wide accuracy is not exclusive. One can achieve both of them when restrictions are put in the frequency in single link measurement and also optimization accuracy is put in global optimization results. However, such dual restriction will lead to unacceptable overhead. In fact, one can achieve the link-wise accuracy through a measurement method [14], which is independent with our compressive sensing method. Our method could provide a promise on the network-wide accuracy that $\beta$ portion of $\left\{p_{i j}^{m}\right\}$ is accurate but leave link-wise accuracy for the implementation.

The accuracy is assured by an adequate number of measurements, while the overhead and time cost metric require as few measurements as possible. Thus, our target is to design the solution that achieves good tradeoffs among these metrics.

2.2. A Linear System Formulation. Basically, the measurement process can be modeled as a linear system. By applying the prevalent compressive sensing [12] theory on this linear system with proper specification, we derive an efficient measurement with low overhead and time cost.

Before introducing the solution with partial measurement and simultaneous measurement, we first formulate our problem in the form of a linear system.

According to the SINR model, the RSS is approximately linear additive. This property implies that when several nodes in the network send signals in the same slot, the RSS of a 
certain node is the sum of the RSSs from all of the sending nodes. Formally, in one time slot, we have $r_{j}=\sum_{i} \phi_{i} p_{i j}$, where $r_{j}$ stands for the RSS measured in node $j$ and $\phi_{i}$ is a binary variable standing for whether node $i$ should send a measurement signal in this time slot. When we extend this formulation into the scenario of multiple time slots and ensemble them into matrix form, we have

$$
\mathbf{R}=\boldsymbol{\Phi} \mathbf{P}
$$

Here, $\mathbf{P}=\left[p_{i j} \mid 0 \leq i, j \leq N\right]$ is called a RSS matrix, whose element $p_{i j}$ represents the RSS from node $i$ to node $j$. The matrix $\mathbf{R} \in \mathbb{R}^{N \times T}$ stands for the measurement result. The element in the $i$ th row and the $j$ th column, denoted as $r_{i j}$, stands for the RSS measured in node $i$ at time slot $j$. According to the SINR model, $r_{i j}$ is the sum of the value of the RSS that node $i$ received. The matrix $\Phi \in\{0,1\}^{T \times N}$, called measurement matrix, stands for the measurement schedule for each node and each time slot, where $\phi_{t j}=1$ indicates that node $j$ sends a measurement signal in slot $t$.

From this linear system perspective, our problem could be stated as follows.

Definition 1 (efficient RSS measurement problem). Given a network of $N$ nodes, try to get the RSS matrix $\mathbf{P}=\left[p_{i j}, \forall i, j \in\right.$ $\{0,1, \ldots, N\}]$ through a planned measurement process $\mathbf{R}=$ $\mathbf{\Phi P}$ with minimum $|\operatorname{row}(\boldsymbol{\Phi})|$.

Note that, if we choose $\Phi$ as an $N \times N$ identity matrix, the RSS matrix could be easily recovered. However, as the column number of $\boldsymbol{\Phi}$ stands for the measurement slots, we need to generate a matrix $\Phi$ with $T \ll N$. Thus, an $N \times N$ identity matrix is unacceptable, especially for the networks with a large number of nodes. The linear system has a unique solution only if $\operatorname{rank}(\Phi)=N$. Thus, a matrix $\Phi$ with $T \ll N$ is not enough for solving $\mathbf{R}=\boldsymbol{\Phi} \mathbf{P}$ with a unique solution. However, this formula could be resolved with the tools provided by compressive sensing theory, as long as $\mathbf{P}$ is sparse enough. The accuracy of recovered $\mathbf{P}$ is assured with high probability.

2.3. Fundamentals of Compressive Sensing. Before presenting our solutions, we briefly introduce the compressive sensing theory. Compressive sensing (or sampling) (CS) $[12,13]$ is a notion generated from the field of signal processing. In the conventional paradigm, natural signals are first acquired at the Nyquist-Shannon sampling rate and then compressed for efficient storage or transmission. CS shifts this paradigm by combining the two processes into a single compressive sampling process, greatly reducing the complexities in data acquisition. The most important idea in CS theory is that a small amount of random linear projections of sparse or compressible signals have contained sufficient information for signal reconstruction and processing.

In other words, signals can be accurately rebuilt based on the following conditions:

(1) The a priori knowledge of sparsity or compressibility of signals is known.
(2) A small number of global linear measurements are provided.

In purpose of integrity and consistency, we present the following definitions.

Definition 2 (sparse signal). Let $d=\left(d_{1}, d_{2}, \ldots, d_{N}\right)^{\top}$ be an $N$-dimensional signal. We say $d$ is a $K$-sparse signal if there are only $K(K \ll N)$ nonzero entries in $d$. Further, we say $d$ is a $K$-sparse signal in $x$ domain, if there exists a set of orthonormal basis, denoted as $\Psi=\left[\psi_{1}, \psi_{2}, \ldots, \psi_{N}\right], \psi_{i} \in$ $\mathbb{R}^{N}$, in which $d$ can be represented by a $K$-sparse vector $x$ :

$$
\begin{aligned}
d & =\sum_{i=0}^{n} \psi_{i} x, \\
\text { or } d & =\Psi x .
\end{aligned}
$$

Compressive sensing theory states that an $\mathrm{N}$-dimensional signal $s$, which is $K$-sparse in the domain of $\Psi(s=\Psi x$, $x$ is a $K$-sparse vector), can be efficiently represented by $T(T<N)$ linearly measurements. Specifically, let $\Phi$ be a $T \times N(T<N)$ matrix; then, the measurements of $s$ can be obtained by $y=\boldsymbol{\Phi} s, s=\boldsymbol{\Psi}$, where $y$ is the measurement results. Matrix $\boldsymbol{\Phi}$ is referred to as measurement matrix and the matrix $\Psi$ is referred to as the representing basis. The key questions are whether it is possible and how to recover the $N$ dimensional signal $s$ from the $T$-dimensional measurements $y$. Candes and Tao [13] have shown that when $K \leq(1 / 2) T$, and $\Phi$ follows the restricted isometry property (RIP) [11], the exact recovery of $d$ can be achieved through solving a linear optimization problem:

$$
\begin{array}{ll}
\min _{x \in \mathbb{R}^{N}} & \|x\|_{l_{1}} \\
\text { s.t. } & y=\boldsymbol{\Phi} s, s=\boldsymbol{\Psi} x .
\end{array}
$$

The $l_{p}$ norm of vector $x$ is defined as $\|x\|_{l_{p}}=$ $\left(\sum_{p=1}^{N}\left|x_{i}\right|^{p}\right)^{1 / p}$. It is known that $l_{1}$-minimization problem can be solved with linear programming (LP) techniques [11]. The $l_{1} / l_{0}$ equivalence relies on the incoherence property [15] between $\Phi$ and $\Psi$ or the restricted isometry property (RIP) [11] of matrix $\boldsymbol{\Phi}=\boldsymbol{\Phi} \Psi$.

It has been established that Gaussian matrix $\Phi \in \mathbb{R}^{M \times N}$, whose entries are independently and identically distributed realizations of certain zero mean random variables with variance $1 / T$, satisfies the RIP with high probability when $T \geq C(K \log (N / K))$, where $C$ is a constant [16].

\section{Compressive Sensing-Based Solution}

As aforementioned, efficient RSS measurement relies on the process of partial measurement and simultaneous measurement. The former could be achieved with solving the linear system modeling, while the latter relies on how we recover the RSS matrix with only a few time slot measurements. Our basic idea is applying the compressive sensing theory to the linear system.

The process of partial measurement and simultaneous measurement is mainly enabled by the careful design of 
measurement matrix in the compressive sensing, which owns the ability to recover the full signal from partially measured signal information and also has the ability to distinguish the overlapped signal when they are linearly combined. We will discuss it specifically when we propose the design of measurement matrix.

3.1. Solution Framework. The success of the solution depends on two crucial components. The first one is the generation of measurement matrix with good RIP. According to the theorem in [17], good RIP refers to that $\delta_{k}$ (restricted isometry constant) is smaller than $\sqrt{2}-1$. Further, we should also find a representation basis, in the space of which the RSS matrix could be represented in the form of $K$-sparse matrix. Note that we deal with a matrix rather than a vector. In the context of matrices, low rank is analogous to sparsity because the spectrum formed by the singular values of a low-rank matrix is sparse. Thus, in our problem, the $K$-sparse matrix means the rank of matrix is $K$.

Assume that the RSS matrix $\mathbf{P}$ is $K$-sparse in a certain domain, which is formally stated as

$$
\mathbf{P}=\Psi \mathbf{P}^{\prime} .
$$

According to (2), the measurement result can be expressed by the product of a matrix and a RSS matrix. Thus, we can rewrite $\mathbf{R}$ by

$$
\mathbf{R}=\Phi \Psi \mathbf{P}^{\prime}
$$

where $\Phi$ is the measurement matrix to be decided. Based on these formalizations, we now proceed to the determination of measurement matrix and representation basis.

3.1.1. Measurement Matrix. In our measurement, the measurement matrix is a binary matrix, with each row as the sending plan of all nodes in one time slot. The number of rows represents the number of time slots used to perform measurements. In addition, because the representation basis is usually orthonormal matrix, the RIP of matrix $\Phi \Psi$ is the same as $\boldsymbol{\Phi}$. Our target is to find a binary matrix, which has good RIP and a small number of rows and fulfill the partial measurement and simultaneous measurement requirements.

To design the measurement matrix satisfying the partial measurement requirement, we only have to make sure the measurement matrix is in form of $\left\{\boldsymbol{\Phi}_{M N}, M<N\right\}$, where $M$ is the row number. In such way, the measurement time cost is smaller than the size of the signal (network size in our case). Thus, this measurement matrix will be satisfied with the partial measurement requirement.

To design the measurement matrix satisfying the simultaneous measurement requirement, we only have to make sure the measurement matrix is in form of $\left\{\boldsymbol{\Phi}_{M N}, \sum_{i} \phi_{i j} \geq 1\right\}$. This implies that each row of the measurement matrix has multiple nonzero entries, which also means there are more than one node performing measurement at the same time. It is worth mentioning that the number of nonzero entries in each row has direct relation with the least number of rows of measurement matrix to ensure an accurate recovery of the
RSS matrix. Specifically, it has to make sure the measurement matrix satisfy the RIP. Thus, by considering both the partial measurement and simultaneous measurement requirements, how to design a good matrix with RIP becomes our major concern. In the following, we present our design.

To begin with, we derive the size of rows for the measurement matrix. This will inherently control the networkwide accuracy. According to [13], $T$ must be larger than $K \log (N / K)$ to provide a recovery accuracy of $1-2 e^{-T \delta / 8}$, where $\delta$ is the restricted isometric constant.

Then, we derive the elements of measurement matrix. As aforementioned, the measurement matrix in CS is usually drawing from a random matrix whose entries are i.i.d. Gaussian variables complying to $\mathcal{N} \sim(0,1 / T)$ [13]. However, due to the randomness in structure and the uncertainty on RIP, these random matrices are prohibited in real applications. In our application, a binary measurement matrix is required. A simple way is to generate a binary matrix with entries complying to Bernoulli distribution with success probability p. According to the proof in [16], this kind of matrix bears good RIP w.h.p.. However, this kind of matrix may have a large portion of nonzero entries, which, in other words, introduces large measurement overheads. To reduce the overhead of measurement, we could apply the result from the work [18]. It provides a binary matrix generated by LPDC (Low Density Parity-Check) whose definition is as follows.

Definition 3 (LDPC matrix [18]). A binary matrix $A(T, N$, d) $\in\{0,1\}^{T \times N}$ consists of $2 \leq d \leq T-2$ nonzero entries per column and $N d / T$ nonzero entries per row. In structure, any two columns are allowed to share at most one same nonzero position.

According to [18], this matrix has good RIP and low density; thus, it greatly fulfills our requirements.

The measurement overhead could be further reduced by a matrix manipulating trick. We split the measurement matrix $\boldsymbol{\Phi}$ into two parts, $\left[\boldsymbol{\Phi}_{1} \mid \boldsymbol{\Phi}_{2}\right]$. Here $\boldsymbol{\Phi}_{1}$ is a $T \times T$ matrix and $\boldsymbol{\Phi}_{2}$ is an $(N-T) \times T$ matrix. In this form, we have the following theorem.

Theorem 4. If matrix $\boldsymbol{\Phi}_{1}$ is unit matrix, and $\boldsymbol{\Phi}_{2}$ has good RIP, then $\left[\Phi_{1} \mid \Phi_{2}\right]$ also has good RIP.

Proof. When $\Phi_{2}$ comply with following equations, we say it has a good RIP:

$$
(1-\delta)\|x\|_{l_{2}}^{2} \leq\left\|\Phi_{2} x\right\|_{l_{2}}^{2} \leq(1+\delta)\|x\|_{l_{2}}^{2}
$$

Then, $\left[\Phi_{1} \mid \Phi_{2}\right]$ is as follows:

$$
\begin{aligned}
\left\|\left[\boldsymbol{\Phi}_{\mathbf{1}} \mid \boldsymbol{\Phi}_{\mathbf{2}}\right] x\right\|_{l_{2}}^{2}=\left\|\left[\boldsymbol{\Phi}_{\mathbf{1}} \mid \boldsymbol{\Phi}_{\mathbf{2}}\right]\left[x_{1}^{T} \mid x_{2}^{T}\right]^{T}\right\|_{l_{2}}^{2} \\
=\left\|\left[\boldsymbol{\Phi}_{\mathbf{1}} x_{1}+\boldsymbol{\Phi}_{\mathbf{2}} x_{2}\right]\right\|_{l_{2}}^{2} \\
=\left\|\boldsymbol{\Phi}_{1} x_{1}\right\|_{l_{2}}^{2}+\left\|\boldsymbol{\Phi}_{2} x_{2}\right\|_{l_{2}}^{2} \\
+2\left\langle\boldsymbol{\Phi}_{1} x_{1}, \boldsymbol{\Phi}_{2} x_{2}\right\rangle .
\end{aligned}
$$


Because $\Phi_{1}$ is unit matrix, we have

$$
\left\langle\Phi_{1} x_{1}, \Phi_{2} x_{2}\right\rangle=\left\langle x_{1}, \Phi_{2} x_{2}\right\rangle=x_{1}^{T} \Phi_{2} x_{2} .
$$

Because $\Phi_{2}$ has good RIP, then we have

$$
\begin{aligned}
& \left|x_{1}^{T} \Phi_{2} x_{2}\right| \leq \delta\left\|x_{1}\right\|_{l_{2}}^{2}\left\|x_{2}\right\|_{l_{2}}^{2} \leq \delta\|x\|_{l_{2}}^{2} \\
& -\delta\|x\|_{l_{2}}^{2} \leq 2 x_{1}^{T} \Phi_{2} x_{2} \leq \delta\|x\|_{l_{2}}^{2} \\
& \left\|x_{1}\right\|_{l_{2}}^{2}+(1+\delta)\left\|x_{2}\right\|_{l_{2}}^{2}=\|x\|_{l_{2}}^{2}+\delta\left\|x_{2}\right\|_{l_{2}}^{2} \\
& \quad \leq(1+\delta)\|x\|_{l_{2}}^{2} \\
& \left\|x_{1}\right\|_{l_{2}}^{2}+(1-\delta)\left\|x_{2}\right\|_{l_{2}}^{2}=\|x\|_{l_{2}}^{2}+\delta\left\|x_{2}\right\|_{l_{2}}^{2} \\
& \geq(1-\delta)\|x\|_{l_{2}}^{2} .
\end{aligned}
$$

Combing (7), (11), (12), and (13), we have

$$
(1-2 \delta)\|x\|_{l_{2}}^{2} \leq\left\|\left[\Phi_{1} \mid \Phi_{2}\right] x\right\|_{l_{2}}^{2} \leq(1+2 \delta)\|x\|_{l_{2}}^{2} .
$$

Then, $\left[\Phi_{1} \mid \Phi_{2}\right]$ has good RIP.

According to this theorem, we find that if we choose an orthonormal matrix as $\Phi_{1}$ and randomly generate $\boldsymbol{\Phi}_{2}$ with good RIP, then, whole matrix $\Phi$ has the same RIP with $\Phi_{2}$. This is due to the orthonormal columns not affecting the RIP of full random matrix. Based on this property, we can choose and identify matrix $\mathbf{I}$ as $\Phi_{1}$, which only has one nonzero element for each column.

3.1.2. Representation Basis. In this section, we describe how to control the sparsity of RSS matrix. Note that, in the RSS matrix, most of the elements are in fact close to but not equal to zero. This situation requires us to carefully drop some elements to make the matrix sparse. With all these considerations, we apply singular value decomposition here. that

Simply stated, a $N \times N$ matrix could be decomposed such

$$
\mathbf{P}=\mathbf{U} \boldsymbol{\Sigma} \mathbf{V}^{\top}
$$

where $\mathbf{U}$ and $\mathbf{V}$ are $N \times N$ unitary matrices (i.e., $\mathbf{U U}^{\top}=$ $\mathbf{U}^{\top} \mathbf{U}=\mathbf{I}$ ), and $\boldsymbol{\Sigma}$ is an $N \times N$ diagonal matrix containing the singular values. The rank of a matrix is the number of linearly independent rows or columns, which equals the number of nonzero singular values of $\boldsymbol{\Sigma}$.

To determine the representation basis $\mathbf{U}$ and $\mathbf{V}$, we could use the path loss model to compute an approximate matrix $\widehat{\mathbf{P}}$. A singular value decomposition performed to $\widehat{\mathbf{P}}$ could help us to get $\mathbf{U}$ and $\mathbf{V}$.

As we learned from several signal propagation models, the RSSs increase linearly with the sending power. The sending power, however, impacts the interference range of the node. In a CS-based solution, we prefer a low-rank RSS matrix. A higher sending power will result in a larger interference and, in turn, make more entries in RSS matrix not close to zero. Meanwhile, if the sending power is tuned too small, the RSSs (which are close to zero) tend to be too vulnerable when encountering noises and recovery errors. Thus, when applying the result derived from too small sending power to the ordinary scenarios, the error ratio will be amplified. A proper sending power is needed for a better performance of the CS-based solutions. According to the experiment result in Figure 7, the sending power should be set to tune the average interference ratio (defined in Section 4.1) smaller than 0.322 .

3.1.3. Recovery of RSS Matrix. Different from traditional work with CS, where the recovery target is a vector, our work aims to recover a matrix with CS. According to [19], the perfect recovery equals solving the following problem:

$$
\begin{array}{ll}
\min & \operatorname{rank}(\mathbf{P}) \\
\text { s.t. } & \mathbf{R}=\boldsymbol{\Phi} \mathbf{P} .
\end{array}
$$

However, the problem is rather hard to solve directly. Thus, in [19], the authors provided an equivalent form of the problem:

$$
\begin{array}{ll}
\min & \|\mathbf{P}\|_{*} \\
\text { s.t. } & \mathbf{R}=\boldsymbol{\Phi P} .
\end{array}
$$

By introducing SVD in (15), we have

$$
\begin{array}{ll}
\min & \left\|\mathbf{P}^{\prime}\right\|_{*} \\
\text { s.t. } & \mathbf{R V}=\boldsymbol{\Phi} \mathbf{U} \mathbf{P}^{\prime} .
\end{array}
$$

Here, $\left\|\mathbf{P}^{\prime}\right\|_{*}$ is the nuclear norm [19]. Thus, the problem is transformed to a convex programming problem and could be solved efficiently. Because $\mathbf{P}^{\prime}$ is in its most low-rank form with the smallest nuclear norm, $\mathbf{U P}^{\prime} \mathbf{V}^{\top}$ will be the original RSS matrix w.h.p.

In summary, the CS-based solution achieves $\mathcal{O}(\log N)$ time efficiency. We summarize the CS-based solution in Algorithm 1.

3.2. Accuracy Control. The accuracy control consists of two parts, namely, controlling the row number of measurement matrix and controlling the sparsity of representation basis.

Regarding the row number of the measurement matrix, it inherently controls the network-wide accuracy. According to [13], $T$ must be larger than $K \log (N / K)$ to provide a recovery accuracy of $1-2 e^{-T \delta / 8}$, where $\delta$ is the restricted isometric constant. Here, the recovery accuracy is related to the measurement time $T$. Thus, with this, we can control the tradeoff between the time consumption and the accuracy.

Regarding the sparsity of the representation basis, the accuracy of the recovery will decrease as we drop some of the small singular values of the original RSS matrix. Thus, to increase the recovery accuracy, the representation basis should comply with the sparsity $K$. The mathematical relation between $K$ and the accuracy will be our future work.

3.3. Dealing with Background Noise. Apart from using the CS solution as a single solution, it could also be used as an 
Require: Position of each node, Number of Nodes

Ensure: RSS matrix $\mathbf{P}_{\mathbf{m}}$ for each channel $m$

(1) Use one node to choose a channel $m$ with less or constant noise.

(2) Utilize the pathloss model to compute an estimation of RSS matrix $\widehat{\mathbf{P}}$;

(3) Decompose $\widehat{\mathbf{P}}$ in to form of $\mathbf{U} \mathbf{\Sigma} \mathbf{V}^{\top}$, using the singular value decomposition method.

(4) Get the sparsity of $\widehat{\mathbf{P}}$ by omitting the singular value smaller than $\delta$;

(5) Generate the measurement matrix $\boldsymbol{\Phi}=\left[\mathbf{I} \mid \Phi_{2}\right]$ following the LDPC matrix with the predetermined time $\operatorname{slot} T=K \log (N / K)$;

(6) Distribute the measurement plan to each node $i$ with $i$ th column of $\Phi$;

(7) Each node sends a test signal following the measurement plan.

(8) The RSS in each node $i$ as $r_{i}$ is aggregating to the central server. Denote as $\mathbf{R}=\left\{r_{1}^{\top}, r_{2}^{\top}, \ldots, r_{N}^{\top}\right\}$;

(9) Solve the problem $\mathbf{R V}=\mathbf{\Phi} \mathbf{U} \mathbf{P}^{\prime}$ with minimizing the $\left\|\mathbf{P}^{\prime}\right\|_{*}$ by linear programming;

(10) Get the RSS matrix $\mathbf{P}_{\mathbf{m}}=\mathbf{U} \mathbf{P}^{\prime} \mathbf{V}^{\top}$

Algorithm 1: CS-based solution.

extension to mitigate the harm due to background noises. In practice, the RSS is not all from the nodes inside network; the background noise is usually sporadic and affects almost all nodes.

The measured result could be divided into two parts: the noise matrix and the RSS matrix. Formally put,

$$
\mathbf{P}=\mathbf{P}_{\mathbf{r}}+\mathbf{P}_{\mathbf{n}}
$$

We could arrange these two matrices as a new one $\mathbf{P}^{\prime}=\left[\mathbf{P}_{\mathbf{r}}\right]$ $\left.\mathbf{P}_{\mathbf{n}}\right]^{\top}$ and form a new measurement matrix as $\boldsymbol{\Phi}^{\prime}=[\boldsymbol{\Phi} \mid \mathbf{I}]$, which is a $T \times 2 N$ matrix. Here $I$ is $T \times N$ identity matrix. Then a CS solution in Algorithm 1 could be performed in the linear system of

$$
\mathbf{R}=\Phi^{\prime} \mathbf{P}^{\prime}
$$

Note that the background noise usually affects a large area of network. The nodes in network could measure the average background noise for each part and derive a low-rank matrix $P_{n}$. In this way, this extension could have the noise and RSS matrix distinguished in a rather low cost.

3.4. Distributed Scheme. The CS solution could be easily transformed into a distributed solution. As each node could easily collect the RSS from the nodes in the networks, assuming the measurement matrix is known, the measurement process in the node $i$ could be formalized as

$$
y_{i}=\Phi x_{i}
$$

where $y_{i}$ is a $T$ vector and $x_{i}$ is an $N$ vector.

Thus, this is a classical compressive sensing form, as long as $x_{i}$ is sparse. To make sure the algorithm is completely distributed, each node should generate the measurement matrix and the representation matrix independently (Algorithm 2).

For the measurement matrix, we can still use the LDPC matrix. As the measurement matrix is a global measurement schedule, it must be synchronized. The random generation could be synchronized for all nodes if they use the same random seed. This seed could be the global clock or the others that have already been synchronized.

For the representation basis, each node could have their own basis without affecting the recovery results. We apply the standard representation basis here. Specifically, we use Discrete Cosine Transform Basis (DCT) [20] here. The RSS vector generally becomes sparse after the DCT transformation.

With the synchronized measurement matrix $\Phi$ and identical representation basis $\boldsymbol{\Psi}_{i}$ we can easily solve $l_{1}$ minimization problem:

$$
\min _{x_{i} \in \mathbb{R}^{N}}\left\|y_{i}-\Phi \Psi_{i} x_{i}\right\|_{l_{1}}
$$

3.5. Implementation Issues. To implement our algorithm to the real deployed wireless networks, we have to consider some implementation issues, especially those with the distributed algorithms. Consequently, in this subsection, we discuss two major issues, time synchronization and measurement matrix generation.

3.5.1. Time Synchronization. Time synchronization has been constantly drawing research attentions ever since the distributed systems to wireless sensor networks and the mac protocol in wireless networks. Our algorithm mainly relies on the network synchronization in two folds. The first one is that our measurements are performed in a slotted fashion, such that the clock must be synchronized among all the nodes. The second one is that the measurement matrices are generated by the same random function with the same seed. This seed is usually the time clock. Thus, the synchronization is critical for our algorithm. The synchronization frequency and the time cost is the major overhead in real implementation. These costs could be alleviated by selecting suitable synchronization scheme. One suitable solution for our situation is the diffusion algorithm proposed in literature [21], which incurs low energy cost and low synchronization delay.

Another critical concern in the synchronization is how to discover the inconsistency of the measurement matrix between each node. This situation is mainly caused by the clock shift. In our algorithm the inconsistency could be easily discovered by neighbor exchange of the measurement matrix or certain form of checksum, for example, the sum of all elements in measurement matrix. 
Require: Number of Nodes, Synchronized global

Ensure: RSS matrix $\mathbf{P}_{\mathbf{m}}$ for each channel $m$

(1) Use one node to choose a channel $m$ with less or constant noise.

(2) Utilize the pathloss model to compute an estimation of RSS matrix $\widehat{\mathbf{P}}$;

(3) Get the sparsity $K$ of $\widehat{\mathbf{P}}$ by omitting the singular value smaller than $\delta$;

(4) Each node generates the measurement matrix $\boldsymbol{\Phi}=\left[\mathbf{I} \mid \boldsymbol{\Phi}_{2}\right]$ following the LDPC matrix with the predetermined time slot $T=K \log (N / K)$ under the random function with the same seed as the global time.

(5) Each node sends a test signal following the measurement plan.

(6) Each node $i$ generate their own representation basis $\Psi_{i}$ using DCT.

(7) Each node solve

and get the RSS in node $i$.

$$
\min _{x_{i} \in \mathbb{R}^{N}}\left\|y_{i}-\Phi \Psi_{i} x_{i}\right\|_{l_{1}},
$$

(8) Aggregate the $x_{i}$ from each node and get the final RSS matrix.

Algorithm 2: Distributed CS-based solution.

3.5.2. Measurement Matrix Generation. As aforementioned, the measurement matrix is generated complying with LDPC matrix. Traditional scheme is to generate a binary matrix with entries complying to Bernoulli distribution with success probability $p$. Both schemes have to resolve the challenge of how to transmit the parameters, for example, $p$ in Bernoulli distribution or $d$ in LDPC matrix. Such information synchronization process could be performed with the clock synchronization concurrently by combining the transmission of the clock and the parameters.

\section{Evaluation}

In this section, we analyze the performance of the proposed solutions with experiments. We first present the experimental methodology and simulation settings; then, we discuss the numerical results.

4.1. Simulation Settings. Our simulations are based on the data collected from the SWIM platform [22]. It consists of 10 wireless nodes, which are capable of running in $802.11 \mathrm{a} / \mathrm{b} / \mathrm{g}$ mode. We collected the data of the RSSI (Receiving Signal Strength Index) of the beacons from each AP. Specifically, we activated one node at a time, while each AP was tuned to 11 different channels sequentially. Then, we walked to 25 different locations (including the locations of $10 \mathrm{AP}$ ), to collect the 50 different beacon messages from one AP in each channel. The RSSI, AP ID, and channel ID were recorded.

We generated several experimental scenarios from this data set. The experimental scenarios consist of 5, 10, 15, 20 different nodes with the RSS between them. We also set the total operating spectrum to approximately $2.4 \mathrm{GHz}$, with 11 channels of $20 \mathrm{MHz}$, which are the general settings in IEEE 802.11g. 200 scenarios are generated to perform a statistical performance comparison evaluation. The throughput of the whole network is computed using the algorithm in [23].

The benchmarks used to quantify our solutions are how the RSS metrics obtained via the solutions impact the performance of the throughput optimization. The accuracy is quantified using the MPE (Mean Percentage Error), which is formally defined as $(1 / N)\left(\left(p_{i j}^{\prime}-p_{i j}\right) / p_{i j}\right)$. Here, $p_{i j}^{\prime}$ is the estimated RSS for $(i, j)$.

Regarding the CS-based solution, we mainly examine how this method improves the performance of SINR-based optimization. We also provide a comparison of the performance between the CS solution and the solution proposed in [14] versus network density. Regarding the network density, we prefer a benchmark that directly connects the network density and the sparsity of RSS matrix. A metric called average interference ratio is used, which stands for how many portions of the network are interfered by a single node. Assuming that $\mathcal{S}_{i}$ stands for the node set interfered by node $i$, we get the formal definition of average interference ratio as $\sum_{i}\left\|\mathcal{S}_{i}\right\| / N^{2}$. In the implementation, we let the row number $T$ of measurement matrix be equal to $K \log (N / K)$. We also examine the performance change of the introduction of the LDPC code generated measurement matrix.

4.2. Experimenting Results. First, we compare the fundamental performance of CS-based solution and model-based solution [14] in terms of their improvement to the SINRbased throughput optimization algorithm. The result in Figure 5 shows that these two solutions performed almost in the same level. They are all close to the optimal results with exhaustive measurement.

We also examine how the recovery is affected by the introduction of LDPC-based measurement matrix. As aforementioned, the LDPC code has better RIP comparing to the Gaussian matrix. It also has the advantage of less measurement cost, as there are limited nonzero elements per row. The latter advantage could be easily examined with mathematical computation. Thus, we do not provide the numerical result here. The experiment examines the recovery advantage of LDPC-based measurement matrix as shown in Figure 6, where the line "CS-w-LDPC" is indeed better than another one. This also implies that better recovery accuracy could help to improve the optimization results.

We further compare these two solutions in different network densities. As mentioned above, dense networks give rise to a RSS matrix with higher rank, which in turn will 


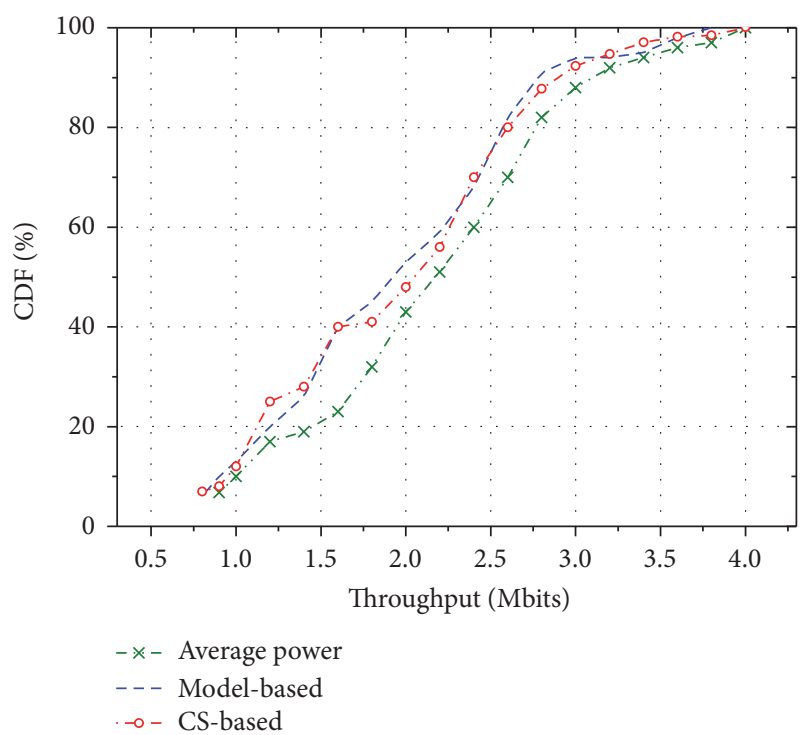

Figure 5: The CDF of throughput under model-based and CS-based solutions.

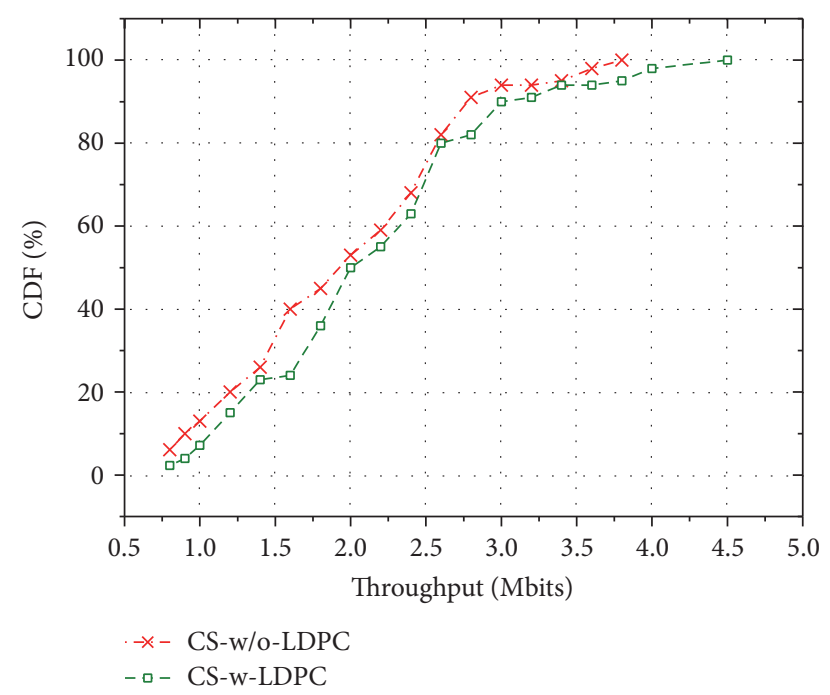

FIGURE 6: The recovery advantage of LDPC-based measurement matrix.

compromise the accuracy. This is illustrated using a MSE versus nodes density graph. In Figure 7, the MPE of the CSbased solution increases with the average interference ratio, while the model-based solution performs stably at different network densities. When the average interference ratio is smaller than 0.3 (which also means $70 \%$ of the RSS matrices are zero entries), the CS-based solution outperforms the model-based solution. When the whole network is a singlehop network, the MPE of the CS-based solution increases to as high as $36 \%$. Thus, the model-based solution is more accurate in the networks whose sending power could not be tuned to reach less than $30 \%$ average interference ratio.

Finally, we examine the time cost of CS-based solution in different network size. The result is shown in Figure 8.

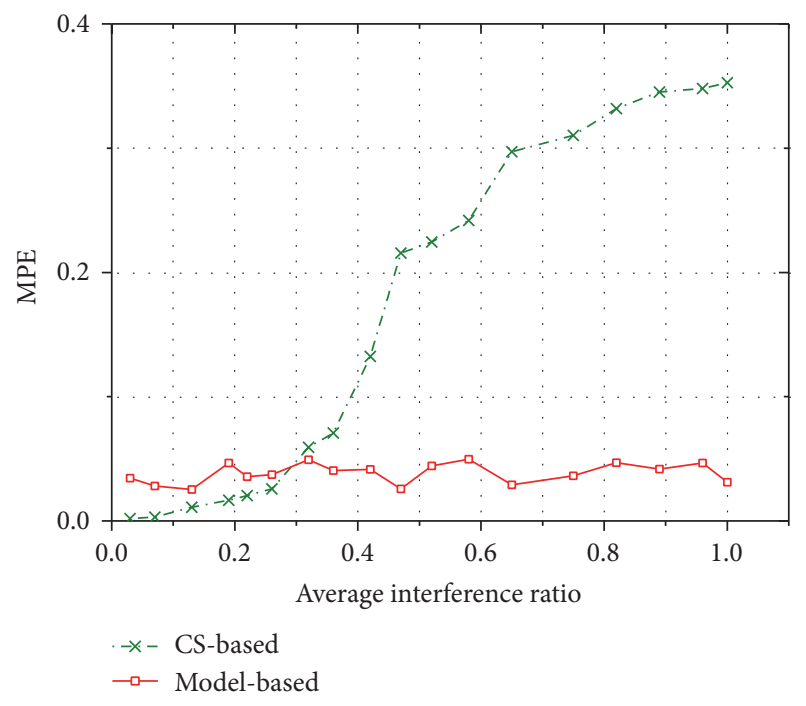

FIGURE 7: The comparison of model-based and CS-based solutions in different network densities.

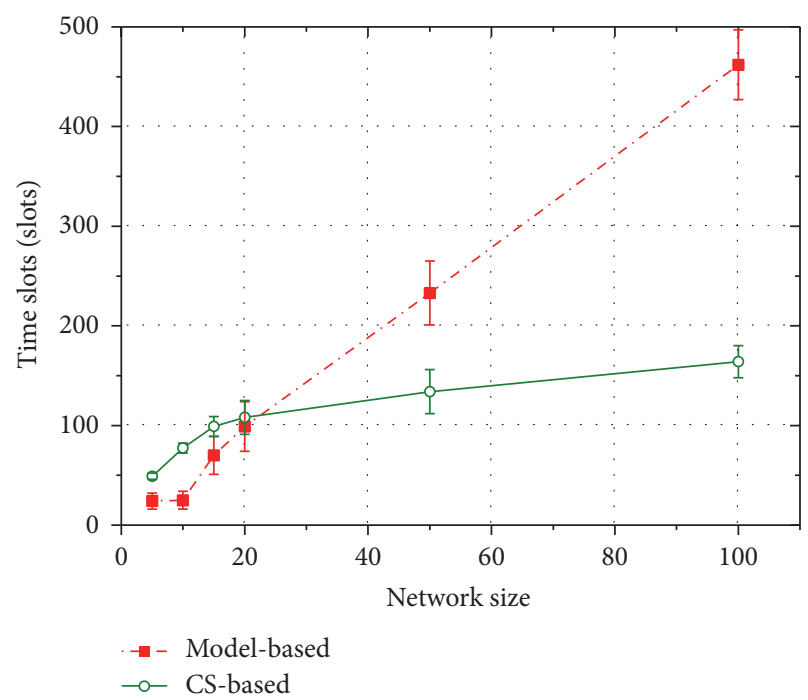

FIGURE 8: The total time slots used by model-based and CS-based solution versus different network sizes.

Note that, to illustrate the advantage of the CS-based solution in a large network size, we generate several 50-nodes and 100 -nodes scenarios. The total channel number is set to 11 . It is clear that the finishing time of the model-based solution grows linearly with network size (except the first two points where the network sizes are small than number of channels), while the CS-based solution grows in logarithm order. The total measurement process for networks with less than 100 nodes takes less than 200 time slots. Today's 802.11 onself products claim 200 500 $\mu$ s per slot. Thus, every round of measurement with accuracy control takes less than one second.

In summary, CS-based solution performed superiorly in the scenario of low density and large network size in terms of time efficiency. 


\section{Related Work}

5.1. RSS Estimation. In [24], the authors considered a network where the path loss between a few low-cost sensors was measured and stored for future use. They proposed an algorithm that employs interpolation techniques to estimate the path loss between a sensor and any arbitrary point in the network. In [25], a PLE estimator based on the method of least squares was introduced in the design of an efficient handover algorithm. Estimation based on a known internode distance probability scheduling was discussed in [26]. The authors assumed that the distance distribution between two neighboring nodes, $i$ and $j$, is known or can be determined easily. Regarding the accuracy control method, in [27], the authors provided a framework to control the accuracy for the measure of the SINR-PRR relation. Our previous work [14] focused on using the path loss model to improve SINR-based optimization in wireless networks.

These works endeavour to make more accurate signal attenuation models in a single transmission. But, none of them focus on making a group measurement on all potential links and all channels, which are crucial for the throughput optimization in wireless networks.

5.2. Applications of Compressive Sensing. Compressive sensing theory was firstly introduced to recover sparse signal with less sampling $[11,13]$, which was latterly found to be useful in compressive data gathering in sensor networks. Compared with the conventional paradigm, CS-based data compression shifts most computations from the encoder to the decoder, which makes it a perfect fit for in-network data processing in wireless sensor networks. The data gathering problem studied immediate data transmission from sensor nodes to a distant base station after data collection. In a single-hop network, compressive wireless sensing (CWS) [28] was shown to be able to reduce the latency of data gathering by delivering linear projections of sensor readings through synchronized amplitude-modulated analog transmissions. Luo et al. [29] explored the compressive data transmission and decoding method, which provided a constant energy consumption scheme. Compressive sensing was also introduced to solve the traffic matrix derivation and interpolation problem [30] and path reconstruction in WSNs [31]. There are some other applications that use the compressive sensing in dealing with cloud media [32]. It mainly considered the changeable property of the wireless network between the media cloud and users and proposed a novel significance-evaluation method for video frames based on CS. Compressive sensing is also applied in the vehicular infotainment system [33]. Our previous paper [34] has explored the efficient RSS measurement algorithm in general wireless networks.

Our method is different from the above method in that our method is used in a distributed fashion and we proposed a new measurement matrix based on LDPC and its variant, which could reduce the measurement cost and time cost greater than the traditional way. Comparing to our previous work, we further consider the feature of the IoT systems and propose distributed algorithm with improved energy efficient measurement matrix.

\section{Conclusion}

The efficiency and accuracy of the RSS measurement in the wireless networks are of great importance for throughput optimization, localization, and wireless sensing in the Internet of Things. Traditional efficient RSS measurement adopt a "measure a few, predict many" fashion with calibrating the parameters in the propagation models. However, we claim that these kinds of methods are not good enough as they miss the chance of simultaneous measurement and controllable partial measurement, which are all achieved with the compressive sensing-based solution we proposed. With CS-based solution, the whole measurement process could be finished in time of $\mathcal{O}(\log N)$, rather than $\mathcal{O}\left(N^{2}\right)$ in the traditional way. Furthermore, an accuracy control tool is also provided to make balance between the accuracy and efficiency in a quantitative way. Experiments with real data trace from our platform have proved the efficiency of our solution.

\section{Conflicts of Interest}

The authors declare that they have no conflicts of interest.

\section{Acknowledgments}

This work is supported in part by the National NSF of China (under Grant nos. 61602238, 61672278, 61672283, and 61373128), the Key Project of Jiangsu Research Program Grant (BK20160805), and the China Postdoctoral Science Foundation (no. 2016M590451).

\section{References}

[1] R. Ahlswede, N. Cai, S. R. Li, and R. W. Yeung, "Network information flow," Institute of Electrical and Electronics Engineers. Transactions on Information Theory, vol. 46, no. 4, pp. 12041216, 2000.

[2] S. Katti, H. Rahul, W. Hu, D. Katabi, M. Médard, and J. Crowcroft, "XORs in the air: Practical wireless network coding," pp. 243-254.

[3] I. F. Akyildiz, W. Lee, M. C. Vuran, and S. Mohanty, "NeXt generation/dynamic spectrum access/cognitive radio wireless networks: a survey," Computer Networks, vol. 50, no. 13, pp. 2127-2159, 2006

[4] N. Nie and C. Comaniciu, "Adaptive channel allocation spectrum etiquette for cognitive radio networks," in Proceedings of the 1st IEEE International Symposium on New Frontiers in Dynamic Spectrum Access Networks (DySPAN '05), pp. 269-278, Baltimore, Md, USA, November 2005.

[5] Y. Zhao, J. Wu, F. Li, and S. Lu, "On maximizing the lifetime of wireless sensor networks using virtual backbone scheduling," IEEE Transactions on Parallel and Distributed Systems, vol. 23, no. 8, pp. 1528-1535, 2012.

[6] Z. Yang, C. Wu, and Y. Liu, "Locating in fingerprint space: wireless indoor localization with little human intervention," in Proceedings of the 18th Annual International Conference on Mobile Computing and Networking (Mobicom '12), pp. 269-280, August 2012. 
[7] W. Wang, A. X. Liu, M. Shahzad, K. Ling, and S. Lu, "Understanding and modeling of WiFi signal based human activity recognition," in Proceedings of the 21st Annual International Conference on Mobile Computing and Networking, MobiCom 2015, pp. 65-76, Paris, France, September 2015.

[8] O. Goussevskaia, Y. A. Oswald, and R. Wattenhofer, "Complexity in geometric SINR," in Proceedings of the 8th ACM International Symposium on Mobile Ad Hoc Networking and Computing (MobiHoc '07), pp. 100-109, September 2007.

[9] R. Bhatia and M. Kodialam, "On power efficient communication over multi-hop wireless networks: joint routing, scheduling and power control," in Proceedings of the 23rd Annual Joint Conference of the IEEE Computer and Communications Societies (INFOCOM '04), vol. 2, pp. 1457-1466, Hong Kong, March 2004.

[10] Y. Shi, Y. T. Hou, S. Kompella, and H. D. Sherali, "Maximizing capacity in multihop cognitive radio networks under the SINR model," IEEE Transactions on Mobile Computing, vol. 10, no. 7, pp. 954-967, 2011.

[11] E. J. Candes and T. Tao, "Decoding by linear programming," Institute of Electrical and Electronics Engineers. Transactions on Information Theory, vol. 51, no. 12, pp. 4203-4215, 2005.

[12] D. L. Donoho, "Compressed sensing," Institute of Electrical and Electronics Engineers. Transactions on Information Theory, vol. 52, no. 4, pp. 1289-1306, 2006.

[13] E. J. Candes and T. Tao, "Near-optimal signal recovery from random projections: universal encoding strategies?" Institute of Electrical and Electronics Engineers. Transactions on Information Theory, vol. 52, no. 12, pp. 5406-5425, 2006.

[14] Y. Zhao, J. Wu, and S. Lu, "Efficient SINR estimating with accuracy control in large scale cognitive radio networks," in Proceedings of the 2011 17th IEEE International Conference on Parallel and Distributed Systems, ICPADS 2011, pp. 549-556, Tainan, Taiwan, December 2011.

[15] E. J. Candès and M. B. Wakin, "An introduction to compressive sampling: a sensing/sampling paradigm that goes against the common knowledge in data acquisition," IEEE Signal Processing Magazine, vol. 25, no. 2, pp. 21-30, 2008.

[16] R. Baraniuk, M. Davenport, R. DeVore, and M. Wakin, "A simple proof of the restricted isometry property for random matrices," Constructive Approximation. An International Journal for Approximations and Expansions, vol. 28, no. 3, pp. 253-263, 2008.

[17] E. J. Candès, "The restricted isometry property and its implications for compressed sensing," Comptes Rendus Mathématique. Académie des Sciences. Paris, vol. 346, no. 9-10, pp. 589-592, 2008.

[18] W. Lu, W. Li, K. Kpalma, and J. Ronsin, "Near-optimal binary compressed sensing matrix," https://arxiv.org/abs/1304.4071.

[19] B. Recht, M. Fazel, and P. A. Parrilo, "Guaranteed minimumrank solutions of linear matrix equations via nuclear norm minimization," SIAM Review, vol. 52, no. 3, pp. 471-501, 2010.

[20] S. A. Khayam, The Discrete Cosine Transform (Dct): Theory and Application, Michigan State University, 2003.

[21] Q. Li and D. Rus, "Global clock synchronization in sensor networks," IEEE Transactions on Computers, vol. 55, no. 2, pp. 214-226, 2006.

[22] http://cs.nju.edu.cn/lwz/swim/swim.html.

[23] Y. Zhao, J. Wu, and S. Lu, "Throughput maximization in cognitive radio based wireless mesh networks," in Proceedings of the 2011 IEEE Military Communications Conference, MILCOM 2011, pp. 260-265, Baltimore, MD, USA, November 2011.
[24] X. Zhao, L. Razoumov, and L. J. Greenstein, "Path loss estimation algorithms and results for rf sensor networks," in Proceedings of IEEE Vehicular Technology Conference (VTC), 2004.

[25] N. Benvenuto and F. Santucci, "A least squares path-loss estimation approach to handover algorithms," IEEE Transactions on Vehicular Technology, vol. 48, no. 2, pp. 437-447, 1999.

[26] G. Mao, B. D. O. Anderson, and B. Fidan, "Path loss exponent estimation for wireless sensor network localization," Computer Networks, vol. 51, no. 10, pp. 2467-2483, 2007.

[27] J. Huang, S. Liu, G. Xing, H. Zhang, J. Wang, and L. Huang, "Accuracy-aware interference modeling and measurement in wireless sensor networks," in Proceedings of the 31st International Conference on Distributed Computing Systems (ICDCS '11), pp. 172-181, Minneapolis, Minn, USA, July 2011.

[28] W. Bajwa, J. Haupt, A. Sayeed, and R. Nowak, "Compressive wireless sensing," in Proceedings of the Fifth International Conference on Information Processing in Sensor Networks, IPSN '06, pp. 134-142, Nashville, Tenn, USA, April 2006.

[29] C. Luo, F. Wu, J. Sun, and C. W. Chen, "Efficient measurement generation and pervasive sparsity for compressive data gathering," IEEE Transactions on Wireless Communications, vol. 9, no. 12, pp. 3728-3738, 2010.

[30] M. Roughan, Y. Zhang, W. Willinger, and L. Qiu, "Spatiotemporal compressive sensing and internet traffic matrices (Extended Version)," IEEE/ACM Transactions on Networking, vol. 20, no. 3, pp. 662-676, 2012.

[31] Z. Liu, Z. Li, M. Li, W. Xing, and D. Lu, "Path reconstruction in dynamic wireless sensor networks using compressive sensing," in Proceedings of the 15th ACM International Symposium on Mobile Ad Hoc Networking and Computing, MobiHoc 2014, pp. 297-306, Philadelphia, Pa, USA, August 2014.

[32] J. Guo, B. Song, and X. Du, "Significance Evaluation of Video Data over Media Cloud Based on Compressed Sensing," IEEE Transactions on Multimedia, vol. 18, no. 7, pp. 1297-1304, 2016.

[33] J. Guo, B. Song, Y. He, F. R. Yu, and M. Sookhak, "A survey on compressed sensing in vehicular infotainment systems," IEEE Communications Surveys \& Tutorials, vol. 99, 2017.

[34] Y. Zhao, W. Li, J. Wu, and S. Lu, "Efficient RSS measurement in wireless networks based on compressive sensing," in Proceedings of the 34th IEEE International Performance Computing and Communications Conference, IPCCC 2015, Nanjing, China, December 2015. 


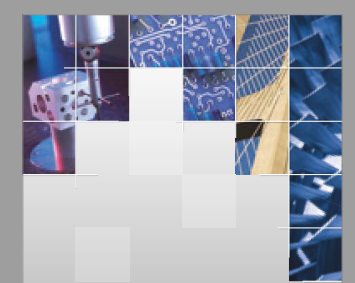

\section{Enfincering}
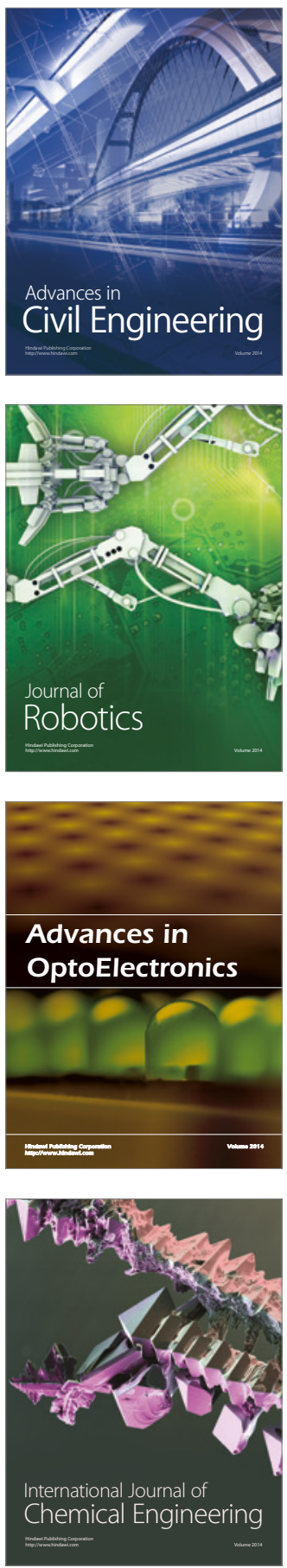

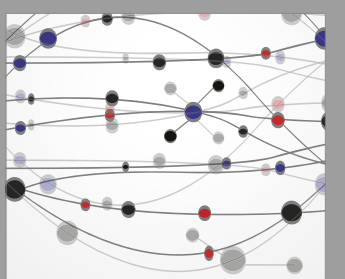

The Scientific World Journal

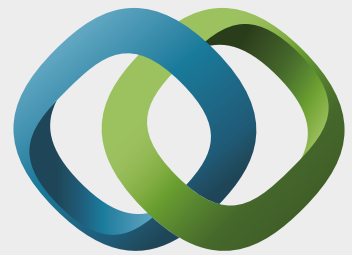

\section{Hindawi}

Submit your manuscripts at

https://www.hindawi.com
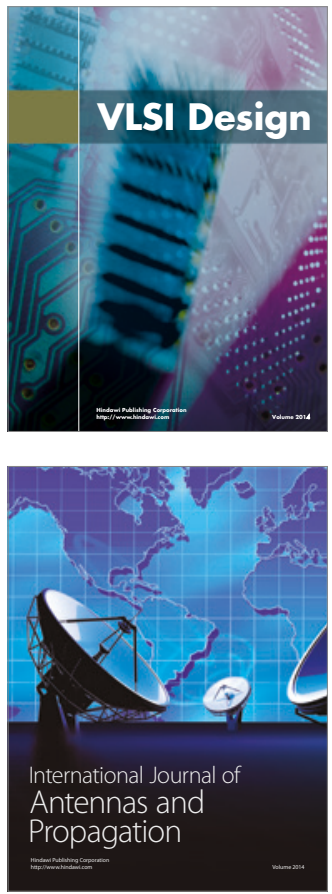

\section{Rotating}

Machinery
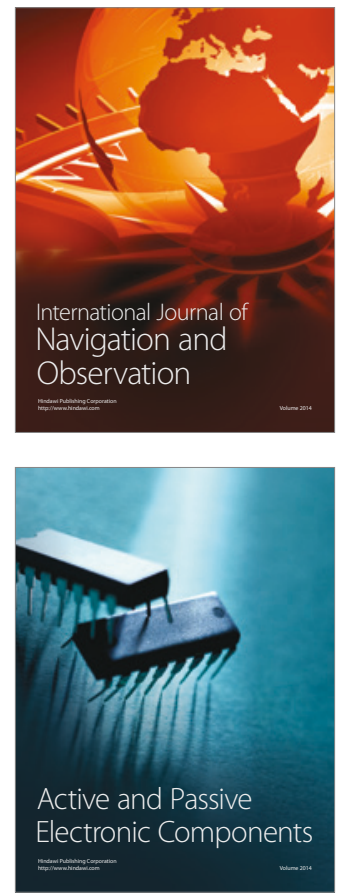
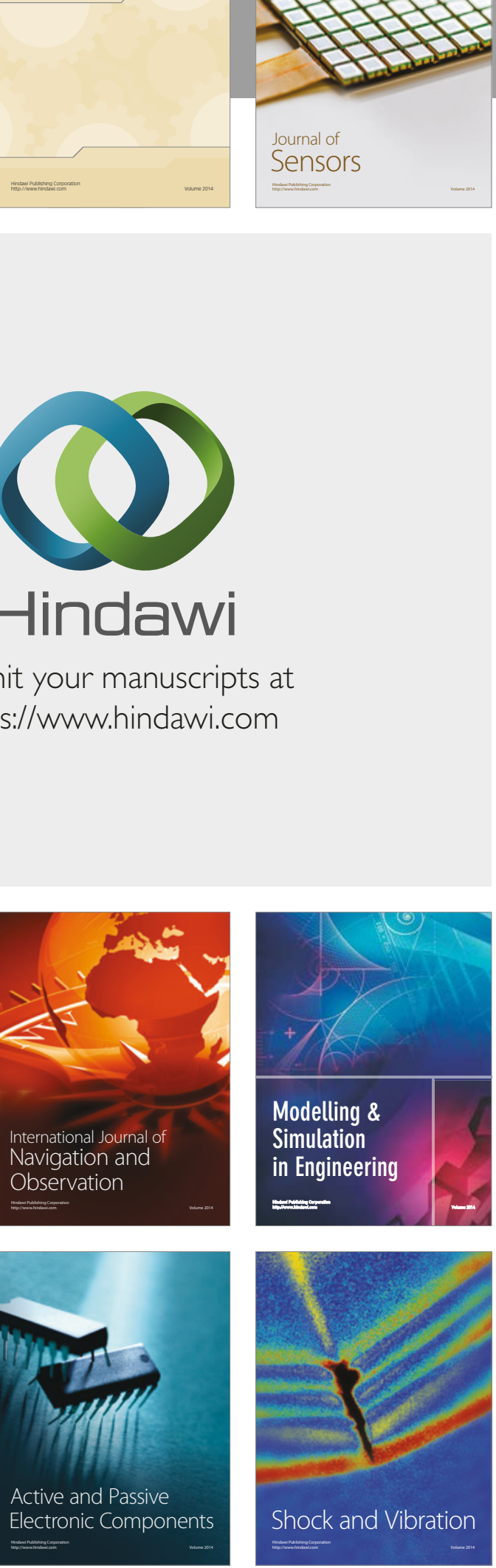
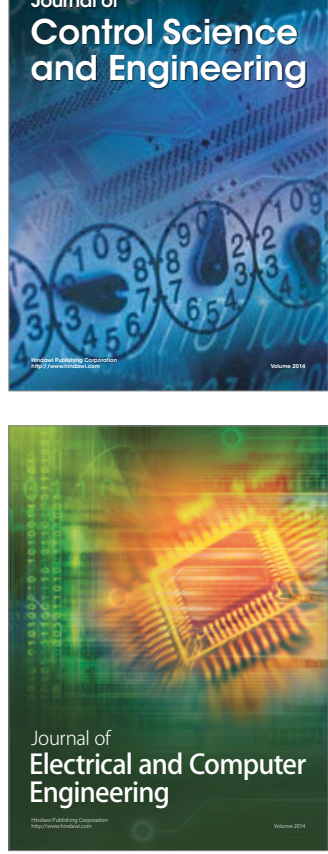

Distributed

Journal of

Control Science

and Engineering
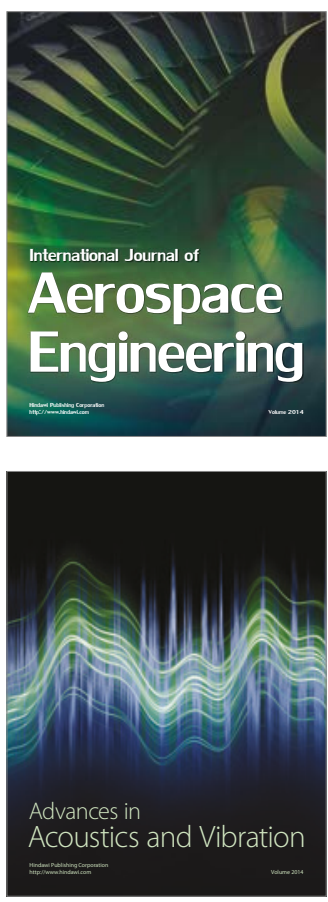

Sensor Networks 